\title{
Theoretical concept and FEM simulations of improved shielding for round horizontal yokes for rotational power loss measurement
}

\author{
Stan Zurek
}

\begin{abstract}
The paper introduces a theoretical concept of a shielding for horizontal yokes for measurements of rotational power loss and other rotational and two-dimensional properties. Apart from horizontal parts, the shielding relies on vertical pieces distributed uniformly around the sample circumference, symmetrically on both sides. The simulations in 2D and 3D FEM show significant improvement in reduction of $H$ perpendicular to the sample surface $\left(H_{z}\right)$. The gradient of the tangential $H$ is reduced so that the extrapolation of values towards the surface might be no longer required. There is also an added benefit that the required magnetomotive force is significantly lower (20-70\%) for achieving the same $B$ in the sample, as compared to the previously used simple horizontal shields.
\end{abstract}

K e y w ord s: 2D FEM, 3D FEM, rotational power loss, magnetic shielding

\section{Introduction}

Rotational power loss in electrical steels is often measured with the use of horizontal magnetising yoke [1]. Such yoke can be made to accept a square sample with two orthogonal magnetising channels in $\mathrm{x}$ and $\mathrm{y}$ directions, similar as shown in Fig. 1(a). The sample of electrical steel can be cut so that the $x$ direction coincides with the rolling and $y$ with the transverse direction (the $z$ direction will be therefore vertical or perpendicular to the surface of the sample).

In a different form, the yoke can be round, also with a two-phase magnetising winding, Fig. 1(b). The sample is then circular and placed horizontally inside the yoke, at its geometrical centre. Other shapes are also possible which can be classified between the two designs, e.g. with three-phase yoke and excitation system.

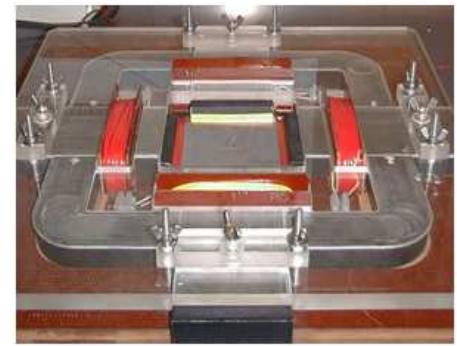

(a)

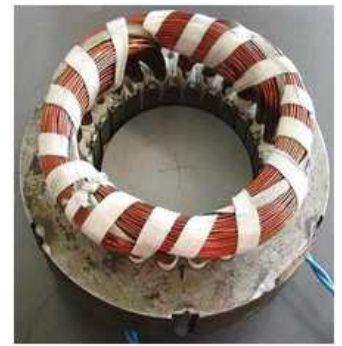

(b)
Fig. 1. Horizontal yoke for rotational loss measurements (a) - for a square sample, (b) - for a circular sample

The geometry of the sample under test and the yoke are such that they result with a significant component of magnetic field strength $H$ perpendicular to the surface of the sample $\left(H_{z}\right)$, as illustrated schematically in Fig. 2.
The $H_{z}$ component arises because there is an air gap between the yoke and the sample. The resulting reluctance between the poles of the yoke and the sample changes only gradually. As a result a significant portion of the magnetic flux enters the sample along any of the magnetic field lines contours (some of them are emphasized as thick grey lines on the left of Fig. 2).

Such distribution of $H$ around the sample presents two difficulties. Firstly, the amplitude of horizontal $H$ increases with the distance from the sample. If $H_{x}$ denotes the component of $H$ parallel to the surface of the sample then a typical distribution along the vertical central line in Fig. 2 would be as shown in Fig. 3. The value $d=0 \mathrm{~mm}$ is the centre of the sample, $d=0.25 \mathrm{~mm}$ is the sample surface, and $d=0.75 \mathrm{~mm}$ corresponds to a point placed $0.5 \mathrm{~mm}$ above the sample surface.

Measurement of $H$ is performed mostly by the assumption of equality of the tangential component of $H$ in the sample, to that just above the surface of the sample [3], which indeed is the case for the data shown in Fig. 3.

However, any $H$ sensor has a finite thickness and cannot measure the field "at the surface" but rather detects a value at some distance $d$ away from the surface. As can be seen in Fig. 3, a distance of $0.5 \mathrm{~mm}$ can lead even to doubling the amplitude of $H_{x}$ to which such sensor would be exposed. The second problem is that the normal component $\left(H_{z}\right)$ can induce significant additional erroneous signals in a single-layer $H$-coil $[4,5]$ or other sensors like strain gauges [6].

One way of overcoming the first problem is to use more than one $H$-coil placed at various distances so that the measured values can be extrapolated to the surface [7,8]. However, the angular positioning of $H$ sensors is known to be critical for reduction of clockwise-anticlockwise discrepancies and an increased number of sensors can easily

formerly Wolfson Centre for Magnetics, Cardiff University, Cardiff, UK, currently with Megger Instruments Ltd, Archcliffe Road, Dover, Kent, CT17 9EN, United Kingdom, stan.zurek@ieee.org 


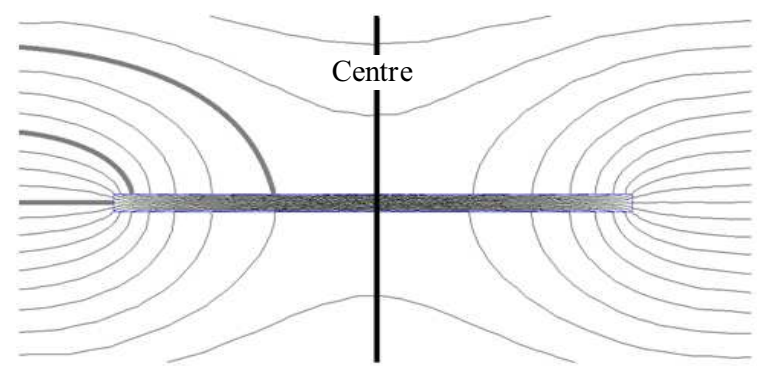

Fig. 2. Magnetic field lines around a sample in horizontal yoke [2]; direction $x$ is parallel and $z$ is perpendicular to the sample surface (drawing not to scale)

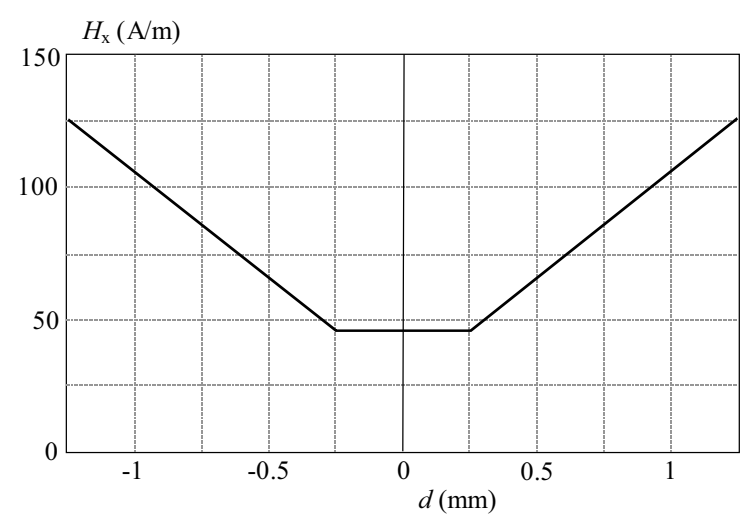

Fig. 3. Amplitude of $H_{x}$ (horizontal $H$ in Fig. 2) increases significantly with the distance from the sample surface [2]; typical data along the line "centre" in Fig. 2.

exacerbate such problems $[9,10]$. This is especially important for rotational fieldmetric measurements which would then require a minimum of six sensors for extrapolation (two for flux density $B$ and four for $H$ ).

Several researchers proposed using shields placed parallel to the sample, so that a "sandwich" arrangement was created with the $H$-coils enclosed between the laminations [11-14].

Experimental and simulation analysis shows that such parallel horizontal shields have limited performance over the whole range of excitation $[2,4,14,15]$. Their effectiveness depends on the distance between the shields and the sample and there is no optimum spacing which would cover the whole range of magnetisation.

This paper presents results of FEM simulation for three different types of systems: without a shield, with parallel horizontal shields and with a new proposed vertical shield, with the same shape of the magnetising yoke.

The proposed new shield shows a qualitative performance comparable or better than the other two configurations.

\section{Definition of configurations}

The cross-section views of the analysed configurations are illustrated in Fig. 4. The control case is the sample without any shields, Fig. 4(a). The yoke is assumed to be of circular shape inside. The sample has $80 \mathrm{~mm}$ diameter (60 $\mathrm{mm}$ was also modelled), with $2 \mathrm{~mm}$ gap on each side between the edge of the sample and the poles of the yoke. The height of the yoke (in the $z$ direction) is $20 \mathrm{~mm}$. The sample and all the shields are $0.5 \mathrm{~mm}$ thick.

All the poles have rectangular endings, without chamfering. It is demonstrated below that the chamfering is not necessary with the new type of the shield.

The second case is the two parallel "horizontal" shields with the same diameter as the sample (Fig. 4b). There is a spacing of $5 \mathrm{~mm}$ between the sample and the shields, on each side. Finally, there is the proposed new configuration with "vertical" shields, Fig. 4(c). The height of the vertical parts is $20 \mathrm{~mm}$ on each side, so that they are wider (taller) than the poles of the yoke.

The small parts of the shields are bent at a right angle so that there is a $10 \mathrm{~mm}$ overlap of a flat part of the shield with the surface of the sample. There is an air gap of $0.1 \mathrm{~mm}$ between the overlapped areas. The overview of the vertical shield is shown in Fig. 5. There are multiple parts, with gaps between them, so the shield does not constitute a full ring. Such gapping is required to significantly increase the reluctance of the shield in the horizontal direction, to encourage the magnetic flux to migrate to the sample, rather than through the otherwise full ring of the shield.

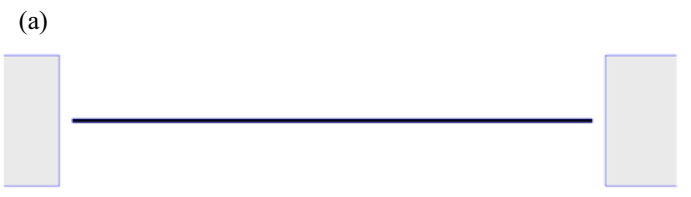

(b)

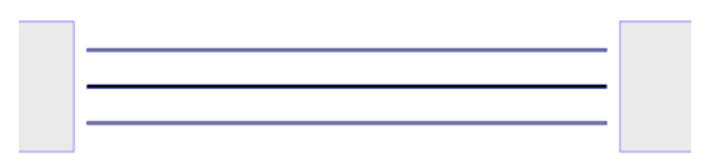

(c)

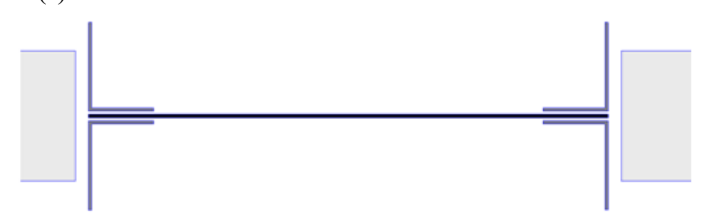

Fig. 4. Cross-section views through the sample - shield configurations: (a) - no shield, (b) - horizontal shields, (c) - vertical shields; black sample, grey shields, light grey magnetic poles of the yoke

For simplicity of modelling it was chosen to represent the yoke as a three-phase structure. However, the poles have concave circular surfaces so they resemble a cylindrical shape, similar to that shown in Fig. 1(b).

There is a square marked on the sample in Fig. 5 and it represents the "uniformly" magnetised area usually used for measurements, $20 \times 20 \mathrm{~mm}$ in this case. 


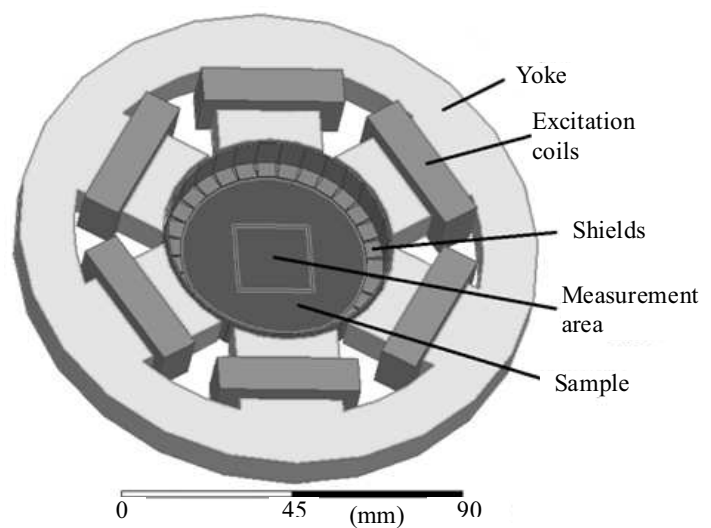

Fig. 5. Top view on the configuration with the horizontal shields, Fig. 4(c)

(a)

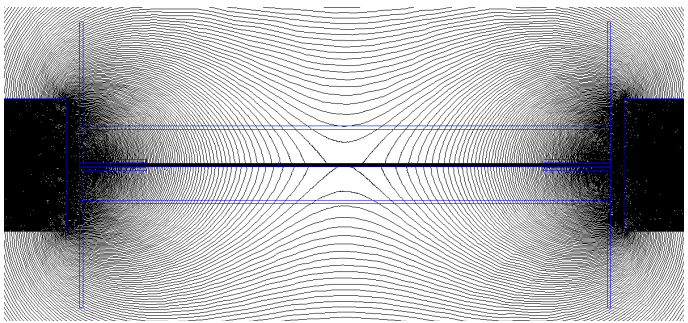

(b)

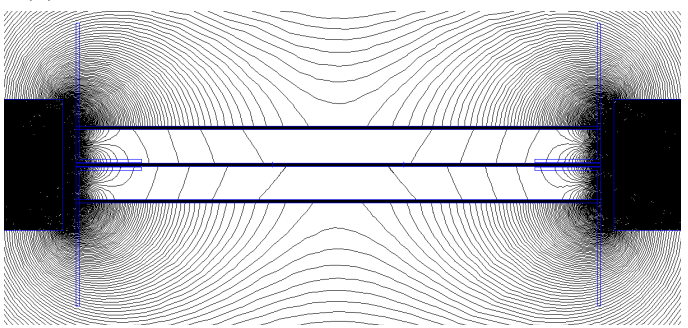

(c)

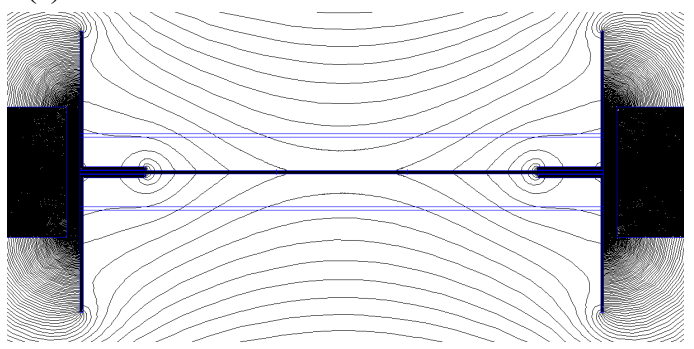

Fig. 6. Magnetic field lines distribution at $1 \mathrm{~T}$ for non - oriented sample and shields : (a) - no shields, (b) - horizontal shields , (c) - vertical shields

\section{2D FEM simulations}

The calculations were performed in 2D [16] and 3D [17] FEM with very similar results from qualitative viewpoint.

In both cases the simulations were carried out with magnetostatic solvers, as these offer better representation of close-to-saturation problems. Magnetostatic solvers represent the non-linear $B-H$ curves in a more accurate way even though hysteresis is not included. The so called "eddy-current" or "time harmonics" solvers by definition assume linear materials even though some provision for non-linearity is made [16,17]. Solutions with saturated materials can lead to non-physical results with such solvers [16]. Nonetheless, as it is shown below, for lower excitation the differences between static and dynamic magnetisations are small from qualitative viewpoint, and hence it is neglected. Mostly 2D results are shown here as they lend themselves to better visualisation and hence much clearer presentation and interpretation for the benefit of the reader. However, some 3D results are also shown for completeness.

The results for $B$ at the centre of the sample adjusted to $1.0 \mathrm{~T}$ are shown in Fig. 6 . All the materials are set to be made from non-oriented steel grade M-27. The contours of all the elements are left in the images in order to show better comparison between all the versions.

At $1.0 \mathrm{~T}$ the horizontal shields indeed give significant improvement over the version without the shields. However, the vertical shields work at least as well, with the number of magnetic field lines reduced around the sample.

The corresponding $H$ values $0.1 \mathrm{~mm}$ above the sample surface for several excitation levels are summarised in Fig. 7.

At $0.3 \mathrm{~T}$ (and below) the vertical shield offers similar performance to the horizontal shields (both curves are on top of each other in Fig. 7(a). At medium to high excitations the vertical shield reduces $H_{z}$ to much lower values than the other two versions.

At even higher excitations the permeability of the sample is reduced due to saturation and the presence or absence of the shield becomes insignificant, because in all cases the $H_{z}$ component is reduced due to the sample saturation anyway, all the $H_{z}$ curves are on top of each other in Fig. 7(d). It should be noted that the data in Fig. 7 also shows the corresponding $H_{x}$ values required to magnetise the sample to the given $B$ level. It interesting to see that up to $1.5 \mathrm{~T}$ (Fig. 7(a),(b),(c)) the amplitude of the $H_{z}$ component within the $20 \times 20 \mathrm{~mm}$ area can be comparable or even significantly exceed the amplitude of the $H_{x}$ component. This is quite significant for the following reason. An $H$-coil is employed to measure only the $H_{x}$ component. $H$-coils might not be completely immune to the perpendicular component and therefore some erroneous signals would be induced $[2,4,5]$.

This is not the case for higher excitations, because the $H_{z}$ component is much smaller in comparison, mostly because the $H_{x}$ component is much greater in order to drive the sample to the required $B$ level (eg $2.0 \mathrm{~T})$.

As mentioned above there is little qualitative difference between DC and AC excitation. A typical comparison is shown in Fig. 8.

Similar qualitative behaviour occurs if the sample and shields are modelled to be made from grain-oriented electrical steel. The results for $1.5 \mathrm{~T}$ are shown in Fig. 9 and they can be easily compared with those for the nonoriented steel in Fig. 6. 
(a)
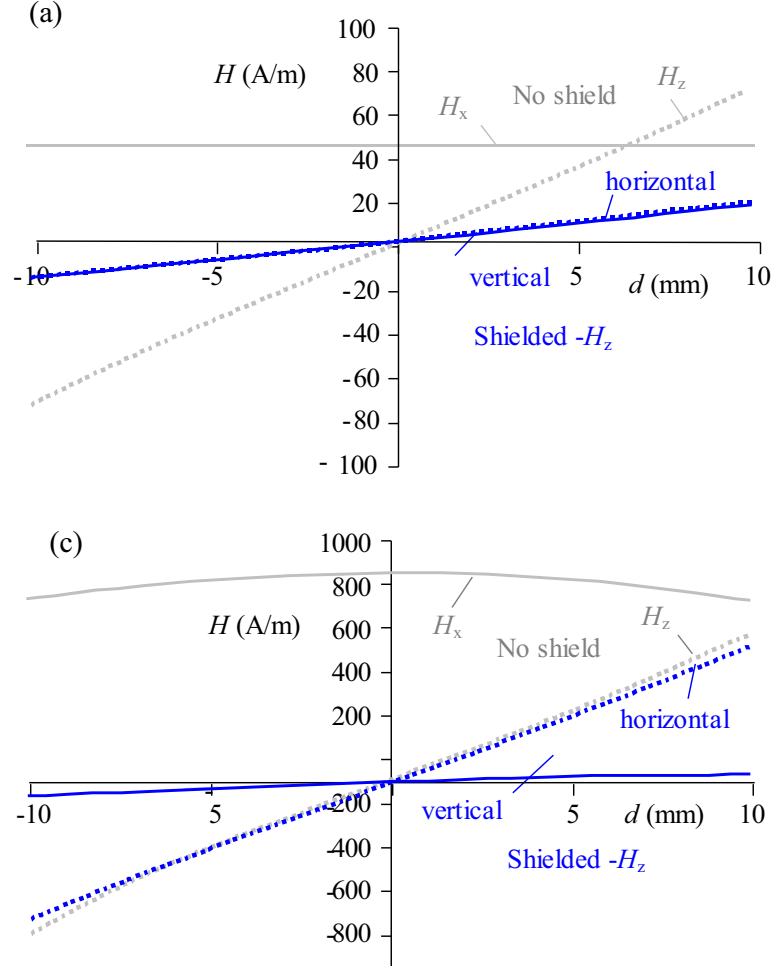

(b)

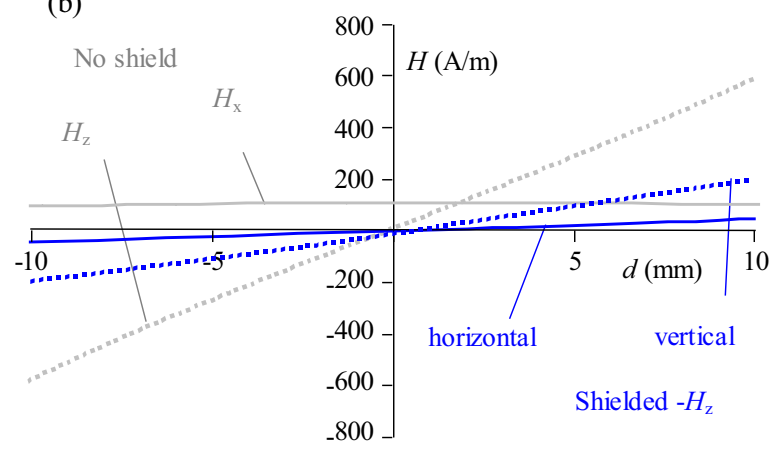

(d)

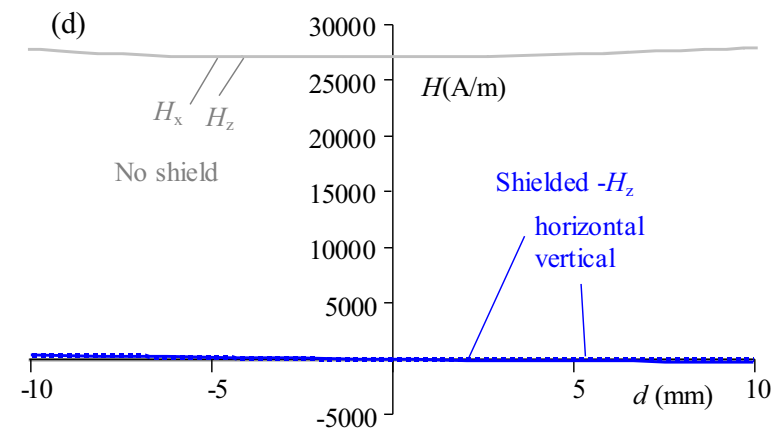

Fig. 7. Distribution of $H_{x}$ and $H_{z}$ for a line $0.1 \mathrm{~mm}$ above the sample surface across $20 \mathrm{~mm}$ distance adjusted to the following $B$ at the centre of the sample: (a) $-0.3 \mathrm{~T},(\mathrm{~b})-1.0 \mathrm{~T},(\mathrm{c})-1.5 \mathrm{~T},(\mathrm{~d})-2.0 \mathrm{~T}$

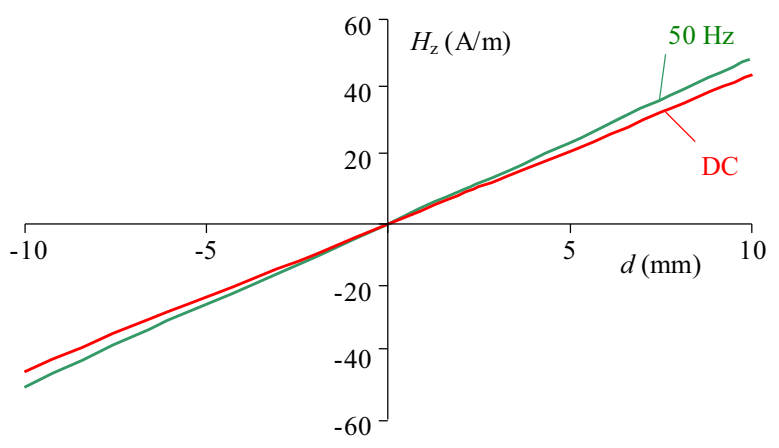

Fig. 8. Comparison of $H_{z}$ distribution for DC and AC excitation, results for vertical shield for sake of brevity

\section{3D FEM simulations}

3D simulations were carried out on a sample with 60 $\mathrm{mm}$ diameter. Qualitatively the 3D results, Fig. 10, reflect those of $2 \mathrm{D}$ simulations.

Again, at very low and very high excitations both types of shielding perform equally well. However, there is a visible improvement for the intermediate levels $(1-$ 1.5 T) where the vertical shielding leads to lower values of $H_{z}$ over the entire width of the measurement area.

For clarity, the $H_{x}$ curve was shown only for the noshield condition to give an idea about the ratio between the magnitudes of $H_{x}$ (required for excitation of the sample) and $H_{z}$ (detrimental for uniform excitation of the sample).

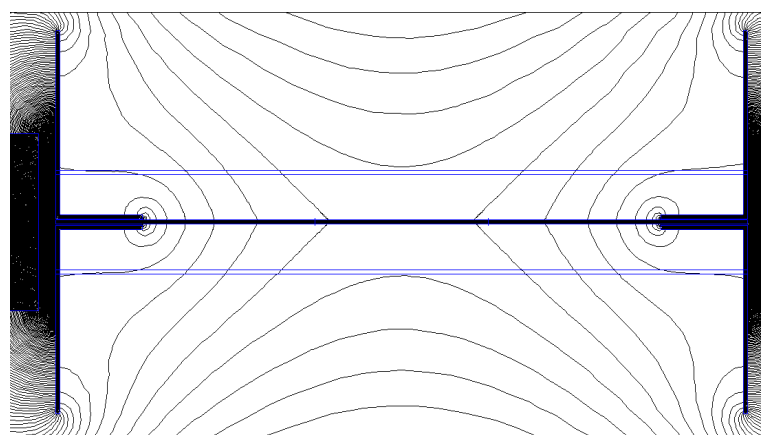

Fig. 9. Sample and shield modelled as grain - oriented electrical steel

The results shown above are only for excitation applied at 0 deg of the $x$ direction. It could be seen from Fig. 5 that the yoke symmetry is repeated every $60 \mathrm{deg}$. So the "worst" angle would be 30 deg which falls between the two neighbouring poles of the yoke. Typical results for 1.5 $\mathrm{T}$ are shown in Fig. 11. The uniformity is at the level of around $1.5 \mathrm{~T} \pm 0.025 \mathrm{~T}(1.7 \%)$ for both cases. Therefore, such vertical shield does not adversely affect the uniformity of excitation.

\section{Other performance factors}

One figure of merit for such shielding could be defined as the ratio of $H_{z}$ to $H_{x}$ amplitudes, or $R=H_{z} / H_{x}$. 
(a)

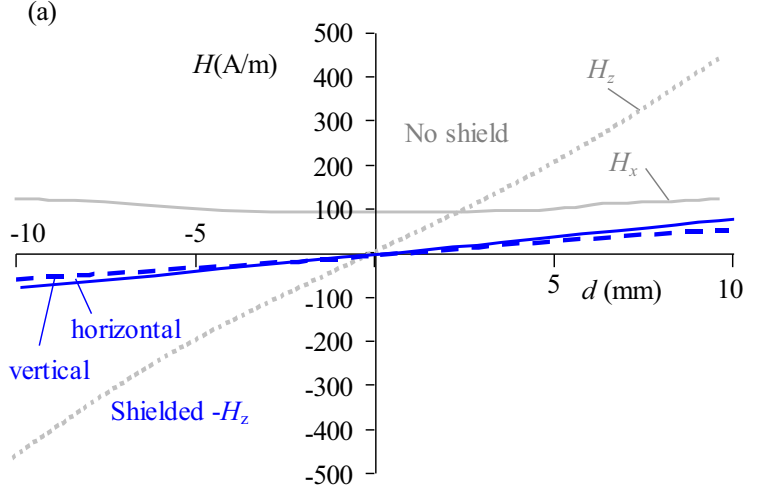

(c)

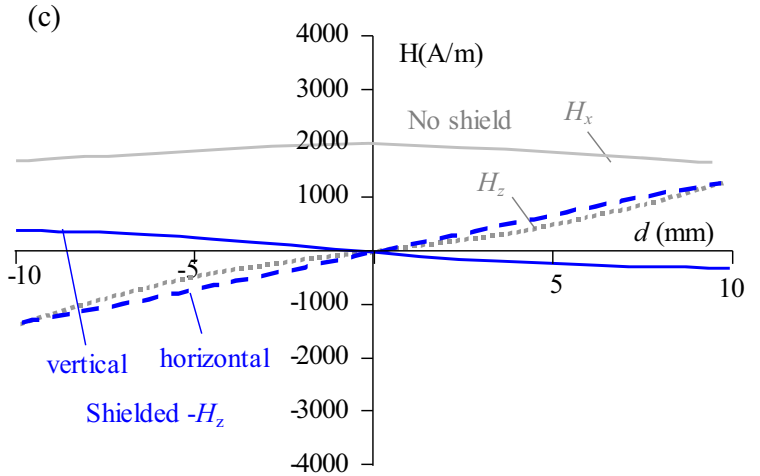

(b)

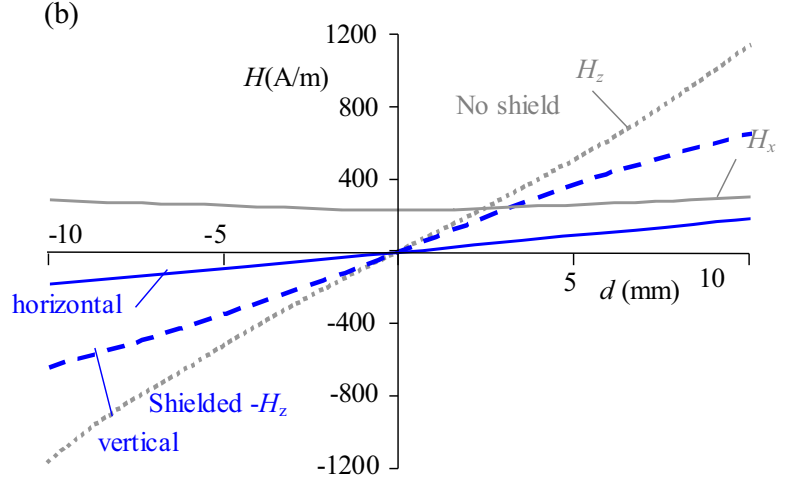

(d)

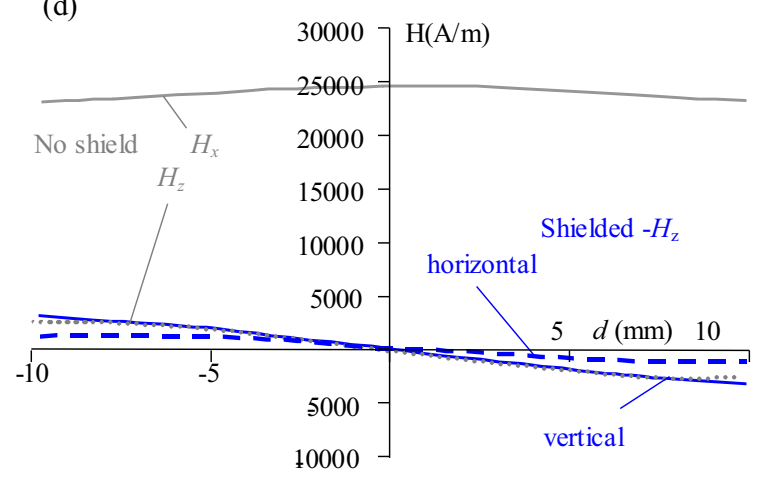

Fig. 10. Results of 3D simulations: (a) $-0.35 \mathrm{~T},(\mathrm{~b})-1 \mathrm{~T},(\mathrm{c})-1.5 \mathrm{~T},(\mathrm{~d})-2 \mathrm{~T}$
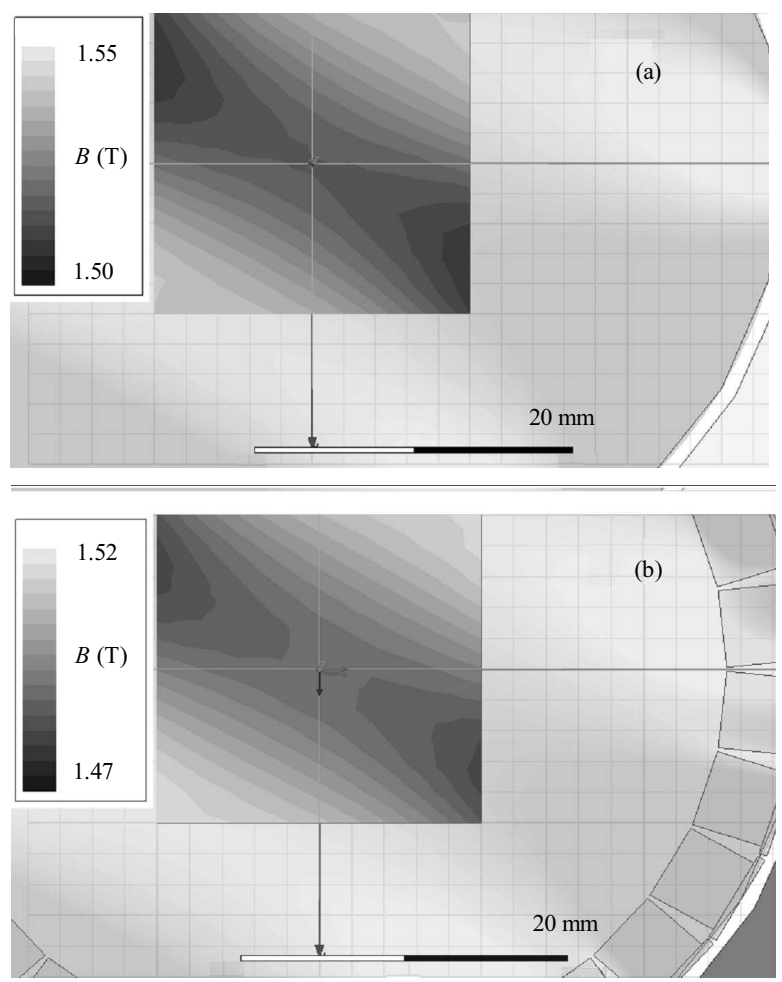

Fig. 11. Distribution of $|\mathrm{B}|$ in the sample at $1.5 \mathrm{~T}$ for 3D FEM: (a) - horizontal shields, (b) - vertical shields

For an ideal shield, the factor $R$ would be always zero so that the unwanted $H_{z}$ component would be negligibly small as compared to the desired $H_{x}$ value.
Such ratios were calculated for the values from Fig. 7 such that the $H_{z}$ value was taken at $d=10 \mathrm{~mm}$ and $H_{z}$ at $d=-0 \mathrm{~mm}$. The resulting curves are shown in Fig. 12. It is evident that the vertical shield provides the best overall performance for all excitation levels.

Interestingly, the configuration with the horizontal shields requires more power or higher magnetomotive force (MMF) to achieve the same excitation level in the sample. This is a consequence of providing alternative paths for the magnetic flux to flow from one pole of the yoke to the opposite, see Fig. 4(b). The flux from the yoke splits into the three alternative paths and the result is that the sample is magnetised to lower $B$ than it is the case without such shields [2], Fig. 4(a). This is not the case for the proposed vertical shields. More of the flux from the magnetic poles is "gathered" by the extending vertical parts, which then guide it into the sample under test. As a result, the sample is magnetised to much higher $B$ for the same MMF applied to the yoke. For $B$ below $1.5 \mathrm{~T}$ the saving is over $70 \%$, but at $2 \mathrm{~T}$ it can still reach $20 \%$, which might be significant for experiments close to saturations.

Of course, the required MMF increases accordingly with the required $B$ to be achieved, but the values were compared to the no-shield case which was assumed to be $100 \%$ for each corresponding $B$ level, Fig. 13 . 
Ratio $R=H_{\mathrm{z}} / H_{\mathrm{x}}$

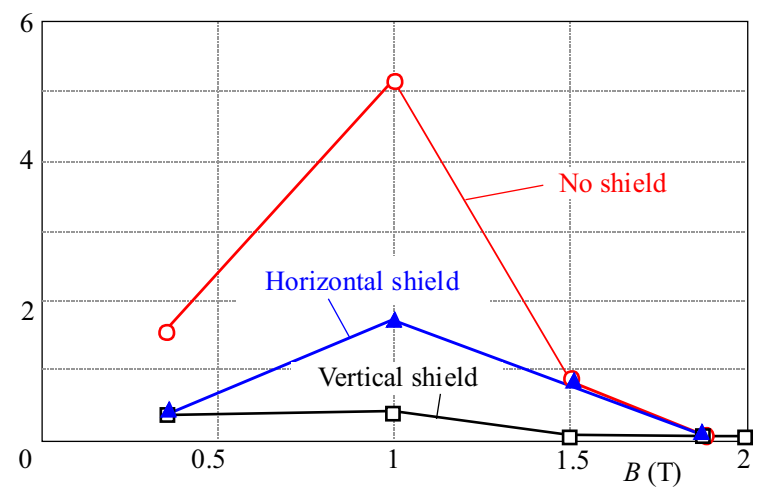

Fig. 12. Ratio $R$ vs excitation level in the sample for all three configurations

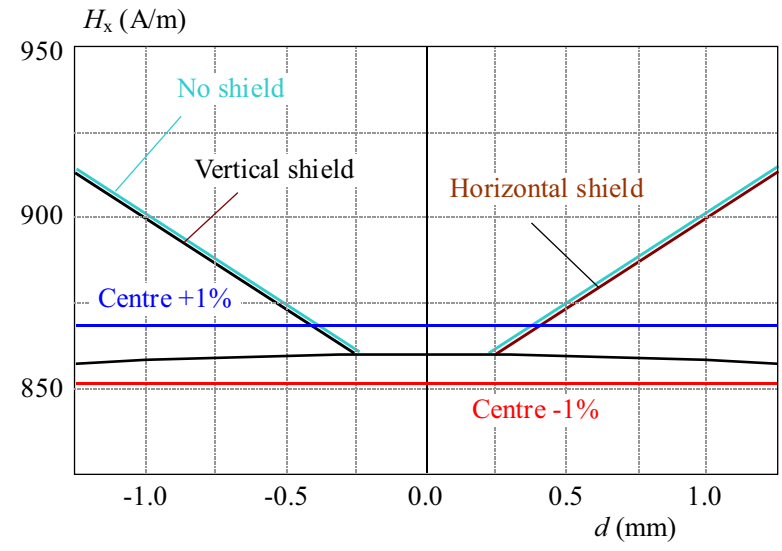

Fig. 14. The gradient of the tangential $H$ component is reduced to better than $\pm 1 \%$ at $1.5 \mathrm{~T}$

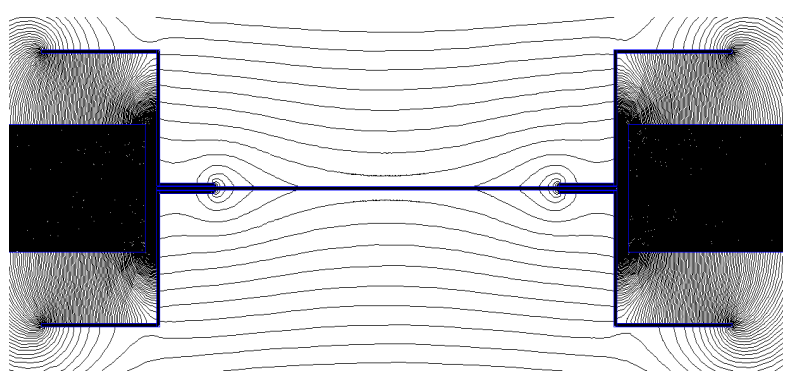

Fig. 15. Further improvement of the vertical shielding

\section{Summary, conclusions and future work}

The aim of this paper was to introduce the concept of the new shield for horizontal yokes. The FEM simulations were used as a tool for assessing the performance as well as producing clear illustrations for description of the idea. The presented results suggest that the proposed shielding configuration is likely to exhibit improved performance at all excitation levels. The yoke-shield reluctance is lowered for the new shield so that more magnetic flux is directed with a greater control. This produces satisfactory $B$ uniformity, reduced $H_{z}$ component and requires lower magnetomotive force for the same $B$.

The gradient of $H$ above the sample is also significantly reduced so that more accurate measurement of the

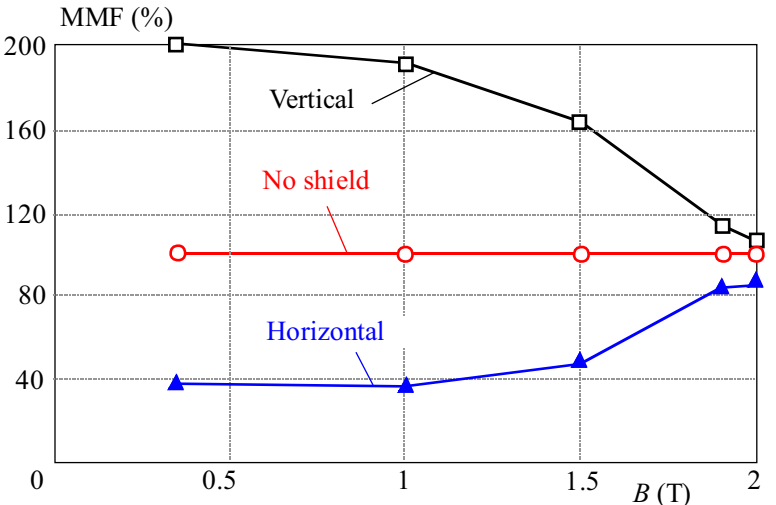

Fig. 13. Required relative MMF vs excitation level

tangential components of $H$ (ie $H_{x}$ and $H_{y}$ ) can be carried out. An example is shown for $1.5 \mathrm{~T}$ in Fig. 14, where the tangential component $\left(H_{x}\right)$ remains within $\pm 1 \%$ of the value inside of the sample. At this level, the performance of the previously used horizontal shield can be similar to the case without any shield. This is because $B$ in the outer horizontal shields is higher than in the sample, so the shields are saturated before the sample [2].

However, it is quite clear that the optimum configuration of such shield is not defined yet, and requires future work, both by simulation and experimental verification.

For instance, one of the improvements could be to extend the vertical parts and wrap them around the poles of the yoke, as shown in Fig. 15. With such configuration, the yoke-shield reluctance is lowered even further, and the $H_{z}$ component is suppressed even more.

For the horizontal shielding, it was suggested in the literature that the shields could be made of the same material as the sample, also if the material was a grain-oriented electrical steel. Therefore, the shields (also referred to as dummy samples) would have the same directional properties (anisotropy) as the sample and the this would help in minimising the $H_{z}$ component because there would be less incentive for the flux to exchange between the sample and the shields.

A comparable concept could be employed for the vertical shield. Namely, each small fragment of the shield, see Fig. 5 and Fig. 11(b) could be made from a material of different reluctance (different permeability or thickness) to correspond to the anisotropy of the sample. Also, all the fragments could be cut at angles corresponding to their angular positioning, so that for instance for each fragment the rolling direction would be aligned with the same direction in the sample.

Of course, the optimum spacing between the vertical shield and the poles of the yoke would have to be investigated for the given sample diameter. The same relates to the size and shape of the "vertical" parts.

However, as shown in this paper the proposed configuration appears to be capable of good shielding performance for all excitation levels even if the optimisation 
was not performed. Moreover, the shield does not limit any access to the central part of the sample, which is important if the $B$ measurement is performed by the needle probe technique. It is expected that if the shield is applied to circular yoke, Fig. (1b), then the performance will be even better, simply because the round yoke is superior in this aspect to the simpler two-phase square magnetisers, Fig. 1(a).

\section{REFERENCES}

[1] J. Sievert et al, "Intercomparison of measurements of magnetic losses electrical sheet steel under rotation flux conditions", Commission of the European Communities, Report EUR 16255 EN, EC Brussels, Luxembourg, 1995.

[2] S. Zurek, "Qualitative 3D FEM study of $B$ and $H$ distribution circular isotropic samples for two-dimensional loss measurements", Electrical Review (Przeglad Elektrotechniczny), R.91, NR 12/2015, 2015, pp. 43.

[3] C. T. A. Johnk,Engineering electromagnetic fields \& waves Willey International Edition New York, USA, 1975, ISBN 9780471442905

[4] S. Zurek, "Sensitivity to off-axis vector components of typical wire wound flat $H$-coil configurations", IEEE Sensors Journal, vol. 17 (13), 2017, pp. 4021.

[5] S. Zurek, "Clockwise-anticlockwise differences of rotational power loss for non-oriented electrical steels due to off-axis $H$-coil sensitivity", under preparation.

[6] S. Somkun, "Magnetostriction and magnetic anisotropy non- oriented electrical steels and stator core laminations", $P h D$ thesis, Wolfson Centre for Magnetics, Cardiff University, Cardiff, UK, 2010.

[7] T. Nakata et al, "Improvement of measuring accuracy of magnetic field strength single sheet testers by using two $H$ coils", IEEE Trans. Magnetics, vol. 23 (5), 1987, pp. 2596.

[8] O. Stupakov et al, "Measurement of electrical steels with direct field determination", IEEE Trans. Magnetics, vol. 46 (2), 2010, pp. 298.

[9] S. Zurek et al, "Errors the power loss measured clockwise and anticlockwise rotational magnetisation. Part 1: Mathematical study", IEE Proceedings, Science, Measurement and Technology, vol. 153 (4), 2006, pp. 147.

10] S. Zurek et al, "Errors the power loss measured clockwise and anticlockwise rotational magnetisation. Part 2: Physical phenomena", IEE Proceedings, Science, Measurement and Technology, vol. 153 (4), 2006, pp .152.

11] D. Makaveev et al, "Measurement system for 2D magnetic properties of electrical steel sheets: design and performance", Proceedings of 6th 1\&2DM Workshop, Bad Gastein, Austria, 2000, pp. 48.

[12] J. G. Zhu et al, "Two dimensional measurement of magnetic field and core loss using a square specimen tester", IEEE Trans. Magnetics, vol. 29 (6), 1993, pp. 2995.

[13] F. Fiorillo et al, "The measurement of rotational losses at IEN", Use of the thermometric method, Proceedings of 1 st $1 \& 2 \mathrm{DM}$ Workshop, Braunschweig, Germany, 1991, pp. 162.

[14] J. Wanjiku et al, "Shielding of the z-component of magnetic field a 2-D magnetizer with a deep yoke", presented at IEEE International Electric Machines \& Drives Conference (IEMDC), 2015.

[15] M. Jesenik et al, "Enlargement of the rotational field homogeneity area a two-phase round rotational single sheet tester", Informacije MIDEM, vol. 32 (2), 2002, pp. 100.

[16] D. Meeker, "Finite Element Method Magnetics", http://www.femm.info.

[16] ANSYS, http://www.ansys.com.

Received 31 March 2017

Stan Zurek graduated at Wolfson Centre for Magnetics, Cardiff University, UK (PhD) and earlier at Czestochowa University of Technology, Poland (MSc). He is a Senior Member of IEEE and an author and co-author of over 80 papers regarding mostly measurements of magnetic properties. He is currently with Megger Instruments Ltd, UK but he continues to study the topic of rotational magnetisation. He is the author of a book titled "Characterisation of soft magnetic materials under rotational magnetisation" published by Taylor \& Francis/CRC. 